\title{
Longitudinal and transverse target-spin asymmetries associated with DVCS on the proton at HERMES
}

\author{
M. Kopytin ${ }^{\mathrm{a}}$ \\ On behalf of the HERMES Collaboration \\ DESY, 15738 Zeuthen, Germany
}

Received: 8 November 2006

Published online: 14 March 2007 - C Società Italiana di Fisica / Springer-Verlag 2007

\begin{abstract}
Measurements of azimuthal cross-section asymmetries from deeply virtual Compton scattering on transversely and longitudinally polarized hydrogen and longitudinally polarized deuterium targets at HERMES are reported. By comparing the HERMES results on the transverse target-spin asymmetry with theoretical calculations based on a phenomenological model of generalized parton distributions, a model-dependent constraint on the total angular momentum carried by quarks in the nucleon is obtained.

PACS. 13.60.-r Photon and charged-lepton interactions with hadrons - 24.85.+p Quarks, gluons, and QCD in nuclei and nuclear processes -13.60. F Elastic and Compton scattering -13.88 . + e Polarization in interactions and scattering
\end{abstract}

\section{Introduction}

Generalized parton distributions (GPDs) were introduced a decade ago as a unified description of hard exclusive processes in the Bjorken regime $[1,2]$. As a generalization of the usual Parton distribution functions (PDFs) they give additional information about quark and gluon properties in the nucleon. Because of their off-forward nature GPDs contain information about both PDFs and nucleon form factors.

Strong interest in GPDs was triggered by the work of Ji [3] who demonstrated that in the forward limit GPDs can give information about the total angular momentum carried by quarks (and gluons) in the nucleon.

The presently cleanest way to access GPDs is to study deeply virtual Compton scattering (DVCS), the hard exclusive electroproduction of a real photon. This process occurs always together with the Bethe-Heitler $(\mathrm{BH})$ process where the photon is radiated from one of the involved leptons. Both processes have identical final states, making them experimentally indistinguishable, and leading to interference between amplitudes.

The transverse target-spin asymmetry (TTSA) associated with DVCS on a transversely polarized target and an unpolarized lepton beam is defined as:

$$
A_{U T}\left(\phi, \phi_{S}\right)=\frac{\mathrm{d} \sigma\left(\phi, \phi_{S}\right)-\mathrm{d} \sigma\left(\phi, \phi_{S}+\pi\right)}{\mathrm{d} \sigma\left(\phi, \phi_{S}\right)+\mathrm{d} \sigma\left(\phi, \phi_{S}+\pi\right)},
$$

where $\phi$ denotes the azimuthal angle between the plane containing the incoming and outgoing lepton momenta

\footnotetext{
${ }^{a}$ e-mail: Mikhail.Kopytin@desy.de
}

and the plane defined by the virtual and the real photons, and $\phi_{S}$ the azimuthal angle of the target polarization vector with respect to the lepton plane. There are two azimuthal amplitudes $A_{U T}^{\sin \left(\phi-\phi_{S}\right) \cos \phi}$ and $A_{U T}^{\cos \left(\phi-\phi_{S}\right) \sin \phi}$ of the TTSA that appear to leading order in $\alpha_{s}$ and $1 / Q$. With the assumption of the dominance of the $\mathrm{BH}$ crosssection w.r.t. that of DVCS, they can be approximated as

$$
\begin{aligned}
A_{U T}^{\sin \left(\phi-\phi_{S}\right) \cos \phi} & \propto \operatorname{Im}\left[F_{2} \mathcal{H}-F_{1} \mathcal{E}\right] \\
A_{U T}^{\cos \left(\phi-\phi_{S}\right) \sin \phi} & \propto \operatorname{Im}\left[F_{2} \widetilde{\mathcal{H}}-F_{1} \xi \widetilde{\mathcal{E}}\right] .
\end{aligned}
$$

Here $\mathcal{H}, \mathcal{E}, \widetilde{\mathcal{H}}$ and $\widetilde{\mathcal{E}}$ denote convolutions of the respective GPDs $H, E, \widetilde{H}$ and $\widetilde{E}$ with hard scattering kernels [4], the skewness parameter is given in the Bjorken limit by $\xi \simeq \frac{x_{B}}{2-x_{B}}$, and $F_{1}$ and $F_{2}$ are the Dirac and Pauli form factors, respectively.

The longitudinal target-spin asymmetry (LTSA) associated with DVCS on a longitudinally polarized target and an unpolarized lepton beam, is defined as

$$
A_{U L}(\phi)=\frac{\sigma^{\Rightarrow}(\phi)-\sigma^{\digamma}(\phi)}{\sigma^{\Rightarrow}(\phi)+\sigma^{\digamma}(\phi)},
$$

where $\Leftarrow(\Rightarrow)$ denotes target spin antiparallel (parallel) to the beam direction. The azimuthal amplitude $A_{U L}^{\sin \phi}$ of the LTSA appears to leading order in $\alpha_{s}$ and $1 / Q$ and can be approximated as

$$
A_{U L}^{\sin \phi} \propto \operatorname{Im}\left[F_{1} \widetilde{\mathcal{H}}+\xi\left(F_{1}+F_{2}\right) \mathcal{H}\right],
$$

with the same notations and assumptions as for eq. (2). 


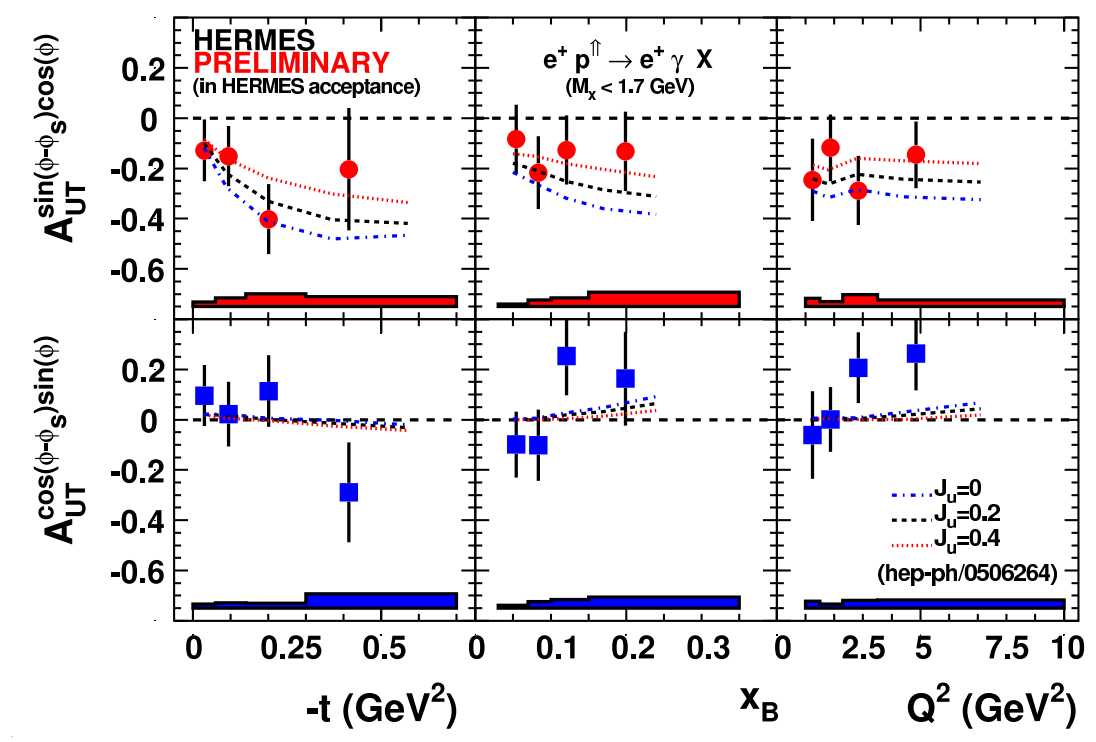

Fig. 1. The $A_{U T}^{\sin \left(\phi-\phi_{S}\right) \cos \phi}$ and $A_{U T}^{\cos \left(\phi-\phi_{S}\right) \sin \phi}$ amplitudes of the transverse target-spin asymmetry associated with DVCS on the proton, shown as a function of $-t, x_{B}$ and $Q^{2}$ for the exclusive sample after background correction. The error bars (bands) represent the statistical (systematic) uncertainties. The curves are the predictions from a GPD model with different $u$-quark total angular momentum $J_{u}$ and fixed $d$-quark total angular momentum $J_{d}=0$ [5].

\section{DVCS at HERMES}

HERMES is a fixed-target experiment using the $27.6 \mathrm{GeV}$ electron or positron beam of HERA [6]. Its internal gas target provides polarized $\mathrm{H}, \mathrm{D},{ }^{3} \mathrm{He}$ as well as unpolarized $\mathrm{H}, \mathrm{D}, \mathrm{Ne}, \mathrm{Kr}$ and Xe targets. In this paper results for $\mathrm{H}$ and $\mathrm{D}$ targets are discussed. HERMES took data with longitudinally polarized hydrogen (deuteron) target in the years 1996-1997 (1999-2000) and a transversely polarized hydrogen target in 2002-2005.

The selected DVCS events are required to have a detected photon in addition to one charged track identified as the scattered lepton. The kinematic requirements imposed on the scattered lepton are $Q^{2}>1 \mathrm{GeV}^{2}$, $W^{2}>8 \mathrm{GeV}^{2}$ and $\nu<23 \mathrm{GeV}$. The polar angle $\theta_{\gamma^{*} \gamma}$ between the virtual and the real photon is required to be between $5 \mathrm{mrad}$ and $45 \mathrm{mrad}$. Since the recoiling proton was not detected the exclusive events were selected with the requirement that the missing mass $M_{x}$ of the reaction $e p \rightarrow e \gamma X$ corresponds to the proton mass. Due to the limited detector resolution the missing-mass range $-1.5<M_{x}<1.7 \mathrm{GeV}$ was selected based on a Monte Carlo simulation for the optimum separation of exclusive events from the semi-inclusive background. The resulting contribution from semi-inclusive $\pi^{0}$ production to the exclusive sample is about $5 \%$. The contribution from the associated exclusive reaction, where the nucleon is excited to a resonant state, amounts to approximately $10 \%$.

\section{Results}

The amplitudes $A_{U T}^{\sin \left(\phi-\phi_{S}\right) \cos \phi}$ and $A_{U T}^{\cos \left(\phi-\phi_{S}\right) \sin \phi}$ of the TTSA as a function of $-t, x_{B}$ and $Q^{2}$, as extracted at HERMES, are shown in fig. 1 [7]. The analysis is based on data accumulated in 2002-2004 with the positron beam and a transversely polarized hydrogen target. Corrections for background and smearing were applied. The main contributions to the systematic uncertainty are those from the determination of the target polarization and the background correction. Also shown in fig. 1 are theoretical predictions based on a phenomenological model of GPDs [8]. The curves represent the TTSA amplitudes evaluated with different $u$-quark total angular momentum $J_{u}$ as a model parameter, while fixing the $d$-quark total angular momentum $J_{d}=0$ [5].

In fig. 2 the dependences of the $A_{U L}^{\sin \phi}$ and $A_{U L}^{\sin 2 \phi}$ amplitudes of the LTSA on proton and deuteron are shown as a function of $-t$ derived from separate fits for every $-t$ bin [9]. A sizable $A_{U L}^{\sin 2 \phi}$ amplitude is found which is expected to be kinematically suppressed w.r.t. $A_{U L}^{\sin \phi}$. It can be sensitive to the next-to-leading contributions to the asymmetry (e.g., twist-3 GPDs $H^{3}, \widetilde{H}^{3}$ ) [4]. No difference is observed between the asymmetries on $\mathrm{H}$ and $\mathrm{D}$ targets in the first $t$ bin, where effects from coherent scattering on the deuteron could be expected. The difference between the two targets in higher $t$ bins might be due to incoherent scattering on the neutron. Calculations based on a GPD model developed in ref. [8] were carried out at the average kinematics of every $t$ bin for the proton. Although the model describes the $A_{U L}^{\sin \phi}$ amplitude well, it does not agree with the data for $A_{U L}^{\sin 2 \phi}$. This can be due to the fact that the twist-3 GPDs are modeled only by the Wandzura-Wilczek term, hence no information on quark-gluon correlations is included.

\section{Constraint on the total angular momentum of quarks in nucleon}

In order to constrain the total angular momentum of quarks a parameterization of GPDs developed in ref. [8] 

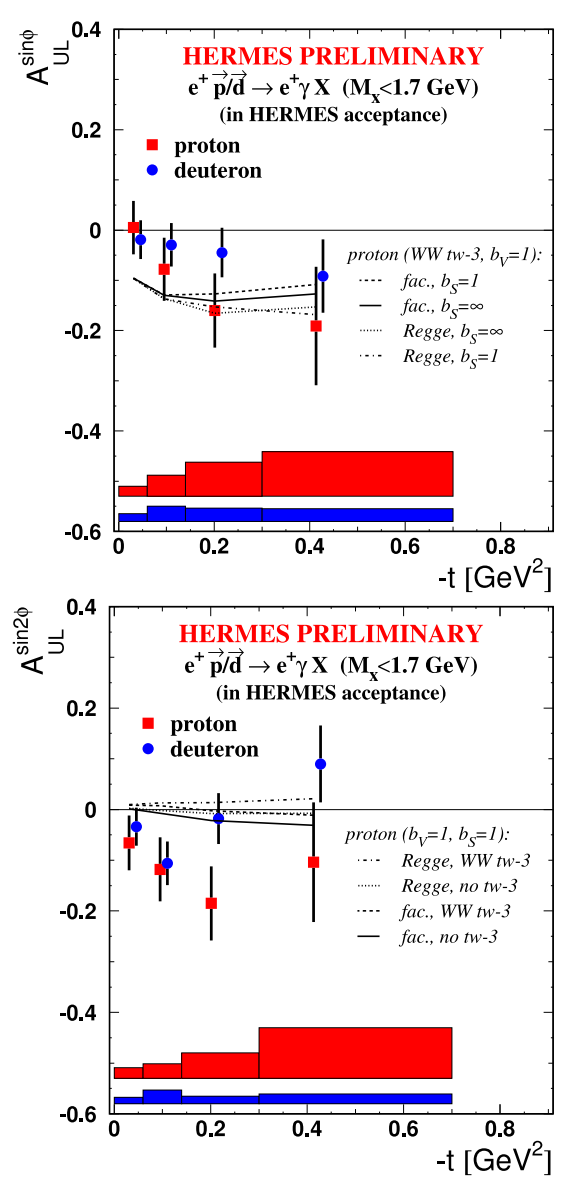

Fig. 2. The $A_{U L}^{\sin \phi}$ (top) and $A_{U L}^{\sin 2 \phi}$ (bottom) amplitudes of the longitudinal target-spin asymmetry on the proton and the deuteron as a function of $-t$. The GPD model calculations for the proton use a factorized or a Regge-inspired $t$-dependence with or without a Wandzura-Wilczek (WW) term [8].

was used where the GPD $E$ is modeled using $J_{u}$ and $J_{d}$ as free parameters. The reduced $\chi^{2}$ value, defined as

$$
\begin{aligned}
& \Delta \chi^{2}=\chi^{2}-\chi_{\text {minimum }}^{2}= \\
& {\left[A^{\text {exp. }}-A^{V G G}\left(J_{u}, J_{d}\right)\right]^{2} /\left[\delta A_{\text {stat. }}^{2}+\delta A_{\text {syst. }}^{2}\right],}
\end{aligned}
$$

is calculated for different values of $J_{u}$ and $J_{d}$. Here $A^{e x p}$. denotes the measured (integrated) TTSA amplitude, $\delta A_{\text {stat. }}\left(\delta A_{\text {syst. }}\right)$ its statistical (systematic) uncertainty, and $A^{V G G}$ are the values calculated at the average kinematics by a code [10] based on the GPD model [8]. As theoretical predictions on $A_{U T}^{\cos \left(\phi-\phi_{S}\right) \sin \phi}$ show minor changes (see fig. 1) with variations in $J_{u}$ and $J_{d}$, only the contribution from $A_{U T}^{\sin \left(\phi-\phi_{S}\right) \cos \phi}$ to the reduced $\chi^{2}$ value is included in eq. (5). The integrated result used in eq. (5) is $\left\langle A_{U T}^{\sin \left(\phi-\phi_{S}\right) \cos \phi}\right\rangle=-0.149 \pm 0.058$ (stat.) \pm 0.033 (syst.), extracted from the 2002-2004 data at the average kinematics $\langle-t\rangle=0.12 \mathrm{GeV}^{2},\left\langle x_{B}\right\rangle=0.095,\left\langle Q^{2}\right\rangle=2.5 \mathrm{GeV}^{2}$.

The area in the $\left(J_{u}, J_{d}\right)$-plane, in which the reduced $\chi^{2}$ value is not larger than unity, is defined as onestandard-deviation constraint on $J_{u}$ vs. $J_{d}$. One obtains $J_{u}+J_{d} / 2.9=0.42 \pm 0.21 \pm 0.06$ (see fig. 3). The first

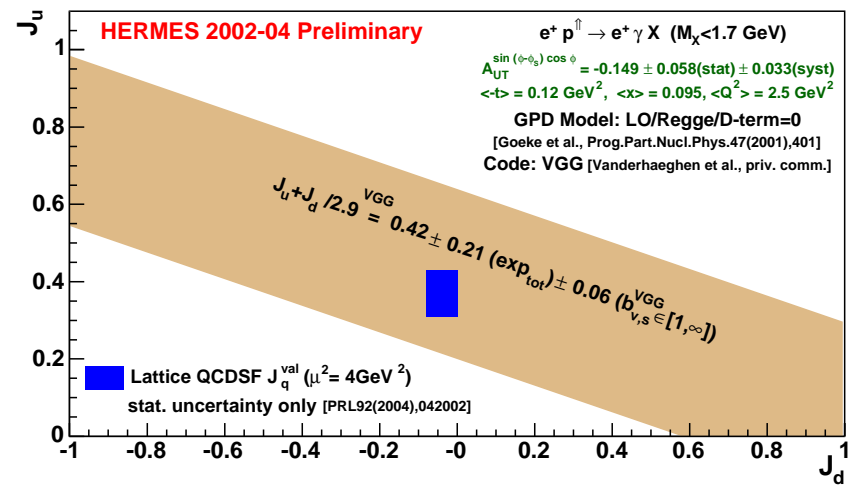

Fig. 3. Model-dependent constraint on $u$-quark total angular momentum $J_{u} v s$. $d$-quark total angular momentum $J_{d}$, obtained by comparing the experimental result and theoretical predictions on the TTSA amplitude $A_{U T}^{\sin \left(\phi-\phi_{S}\right) \cos \phi}$ [11]. Also shown is a lattice result from the QCDSF Collaboration, obtained at the scale $\mu^{2}=4 \mathrm{GeV}^{2}$ for valence quark contributions only.

uncertainty is due to the experimental uncertainty in the measured TTSA amplitude. The second one is a model uncertainty, obtained by varying from unity to infinity the unknown profile parameter $b$ which controls the skewness of GPDs [8]. The $t$-dependence of GPDs is modeled using the Regge ansatz [8]. The impact of using it or its alternative - the factorized ansatz - on the theoretical prediction on the TTSA amplitudes was found to be negligible [5]. The D-term contribution to the GPDs $H$ and $E$ is set to zero, as suggested by HERMES results on the beam charge asymmetry [12]. If the D-term is modeled according to the chiral quark soliton model [8], the resulting constraint is $J_{u}+J_{d} / 2.9=0.53 \pm 0.21 \pm 0.06$.

\section{References}

1. D. Mueller et al., Fortschr. Phys. 42, 101 (1994) hep-ph/9812448.

2. A. Radyushkin, Phys. Lett. B 385, 333 (1996) hep-ph/9605431.

3. X. Ji, Phys. Rev. Lett. 78, 610 (1997) hep-ph/9603249.

4. A. Belitsky, D. Müller, A. Kirchner, Nucl. Phys. B 629, 323 (2002) hep-ph/0112108.

5. F. Ellinghaus, W.D. Nowak, A.V. Vinnikov, Z. Ye, Eur. Phys. J. C 46, 729 (2006) hep-ph/0506264.

6. HERMES Collaboration (K. Ackerstaff et al.), Nucl. Instrum. Methods A 417, 230 (1998) hep-ex/9806008.

7. HERMES Collaboration (Z. Ye), PoS (HEP2005) 120 (2006) hep-ex/0512010.

8. K. Goeke, M. Polyakov, M. Vanderhaegen, Prog. Part. Nucl. Phys. 47, 401 (2001) hep-ph/0106012.

9. HERMES Collaboration (M. Kopytin), AIP Conf. Proc. 792, 424 (2005).

10. M. Vanderhaeghen, P. Guichon, M. Guidal, Computer code for the calculation of $D V C S$ and $B H$ processes in the reaction ep $\rightarrow e p \gamma$ (2001), private communication.

11. HERMES Collaboration (Z. Ye), hep-ex/0606061 (2006).

12. HERMES Collaboration (A. Airapetian et al.), hep-ex/0605108 (2006). 